\title{
6 Melanoma Helper Peptide Vaccine
}

National Cancer Institute

\section{Source}

National Cancer Institute. 6 Melanoma Helper Peptide Vaccine. NCI Thesaurus. Code C153515.

A multi-epitope vaccine containing the following six class II MHC-restricted peptides: gp100, MelanA/MART -1, two tyrosinase peptides, and the cancer/testis antigens MAGE$A 3$ and MAGE-A1,2,3,6, with potential antineoplastic activity. Upon administration, melanoma helper peptides induce an antigen-specific, Th1-dominant, CD4+ T-cell response, potentially augmenting cytotoxic $\mathrm{T}$-cell $(\mathrm{CT} \mathrm{L})$ responses and maintaining immunologic memory against tumor cells expressing melanoma-specific antigens. The $6 \mathrm{MHP}$ vaccine may also induce a CD8+ T-cell response through epitope spreading, potentially priming subsequent immune responses against tumor cells. 